\title{
The Sendai declaration for the eradication of kidney disease
}

\author{
Naoki Kashihara ${ }^{1} \cdot$ Masaomi Nangaku $^{2} \cdot$ Sadayoshi to $^{3} \cdot$ Akira Nishiyama $^{4} \cdot$ David Harris $^{5}$
}

Published online: 25 January 2018

(c) Japanese Society of Nephrology 2018

When kidney disease progresses, it may lead to not only kidney failure but also to diseases such as stroke and myocardial infarction. As such, it has been recognized as a serious disease for its potential impairment of a healthy life. Advancing age tends to contribute to the disease. Indeed, with the swelling of our aging society, the number of dialysis patients continues to increase every year. Consequently, the increasing numbers put pressure on available medical finances. It is necessary to take effective measures against kidney disease quickly. The measures in place are ineffective, despite efforts on a global scale.

Considering all this, the International Society of Nephrology and the Japanese Society of Nephrology announced the Sendai Declaration on May 27, 2017, during the 60th Annual Meeting of the Japanese Society of Nephrology (Fig. 1). The Sendai Declaration is a joint announcement of the intention to combine the strengths of the International Society of Nephrology and Japanese Society of Nephrology to advance knowledge worldwide towards the eradication of kidney disease and to promote all types of related activities that facilitate the spread of awareness, diagnosis, and treatment of kidney disease. We are pleased that the Declaration was signed by three leaders in the realm of kidney health, namely Japanese Society of Nephrology President Naoki

The editorial has been originally published in the Japanese Journal of Nephrology (2017; 59(8): 1090-1091) in Japanese. This is the English version of the article.

Naoki Kashihara

office@jsn.or.jp

1 Japanese Society of Nephrology, Nichinai Hall, 2-38-8 Hongo, Bunkyo-ku, Tokyo 113-0033, Japan

2 Committee on International Relations, Japanese Society of Nephrology, Tokyo, Japan

3 60th Annual Meeting of the Japanese Society of Nephrology, Tokyo, Japan

4 Committee on International Relations, Japanese Society of Nephrology, Tokyo, Japan

5 International Society of Nephrology, Brussels, Belgium
Kashihara, 60th Annual Meeting of the Japanese Society of Nephrology President Sadayoshi Ito, and International Society of Nephrology President David Harris, during a ceremony at the 60th Annual Meeting of the Japanese Society of Nephrology.

The following are the actual contents of the Sendai Declaration (Fig. 2).

The International Society of Nephrology and Japanese Society of Nephrology here declare that, in the future, we will cooperate to pursue the following activities in earnest:

- Continue to work constantly to make the public understand the importance of early detection and early treatment of kidney disease.

- Promote examinations to detect kidney disease at an early stage.

- Develop education and training materials concerning kidney disease on a global scale.

- Aim to reduce the number of patients with kidney disease and related diseases.

The International Society of Nephrology and Japanese Society of Nephrology here declare that, in the future, we will cooperate to support the following activities:

- Promotion of the latest cutting-edge research for the purpose of clarifying the mechanisms of kidney disease.

- Creation of evidence-based guidelines for the prevention, diagnosis, and management of kidney disease.

- Personalization of patient care based on guidelines for the purpose of presenting the most appropriate medical care.

- Establishment of a holistic medical care system for kidney disease.

The Japanese Society of Nephrology, based on the Sendai Declaration, will cooperate with the International Society of Nephrology to fight an unceasing battle to provide new hope for the eradication of kidney disease, by globally promoting all forms of relevant activities, such as those related to 


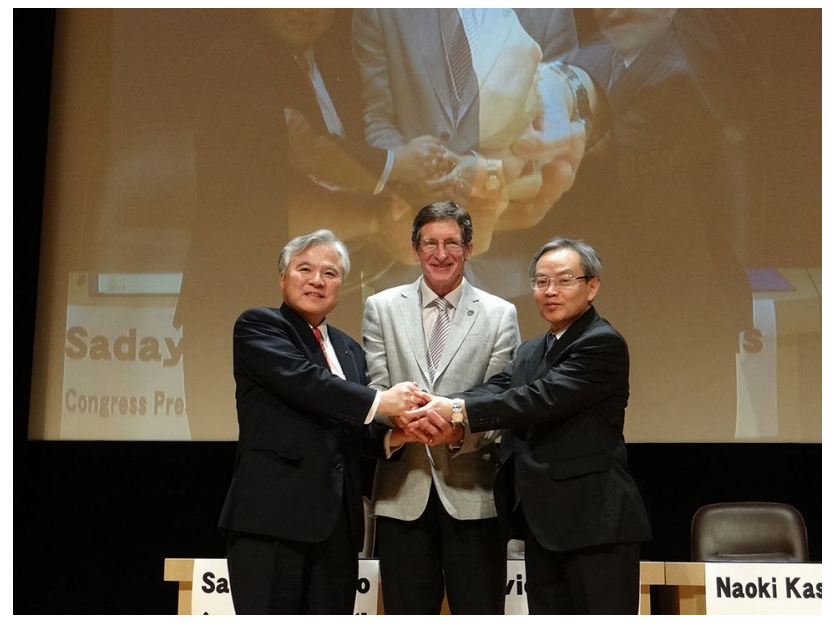

Fig. 1 Ceremony photo of the Sendai declaration

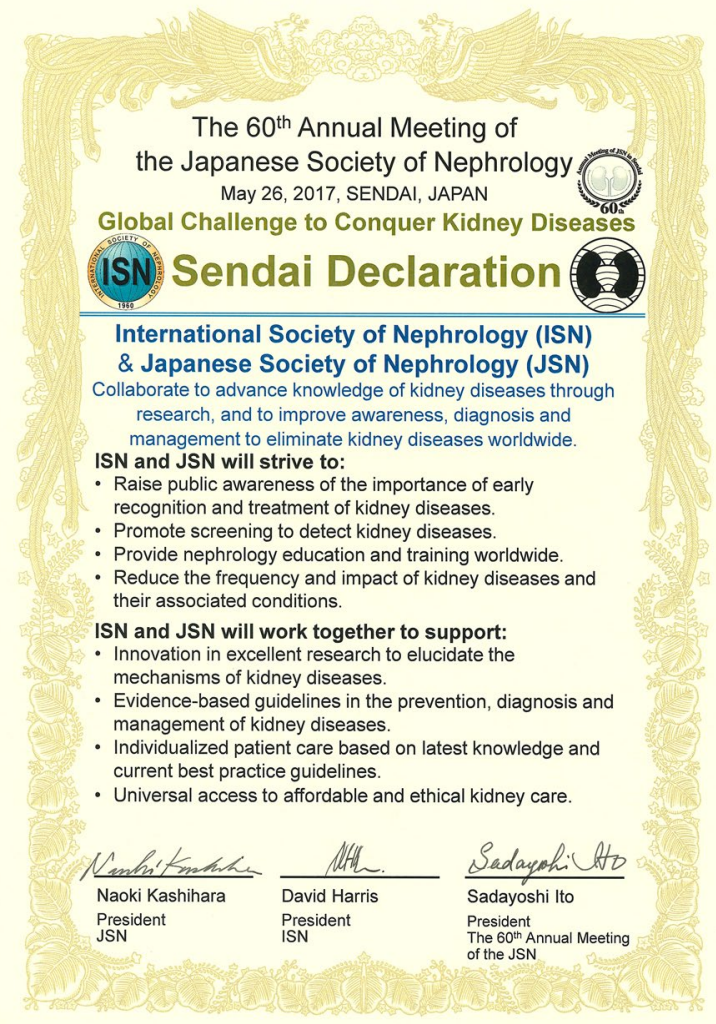

Fig. 2 Sendai declaration kidney disease education, prevention, and treatment. We will also promote the internationalization of the Japanese Society of Nephrology, advance the achievement of a standard of care on a global scale, and play a leadership role in Asia in promoting international cooperation. Currently, specific measures are being studied, a number of which are already being implemented. We sincerely ask for your understanding and cooperation. 